\section{(C) OPEN ACCESS}

- Additional material is published online only. To view please visit the journal online (http://dx.doi.org/10.1136/ thoraxjnl-2017-210326).

For numbered affiliations see end of article.

\section{Correspondence to} Professor Yong Chul Lee, Department of Internal Medicine, Research Center for Pulmonary Disorders, Chonbuk National University Medical School, Jeonju 561-180, South Korea; leeyc@jbnu.ac.kr

JSJ, KBL and SRK contributed equally.

Some of our data have been previously presented in the form of an abstract at an international scientific meeting (the 22nd congress of the Asia Pacific Society of Respirology, 2017).

Received 28 March 2017 Revised 20 February 2018 Accepted 26 March 2018 Published Online First 5 April 2018
Check for updates

To cite: Jeong JS, Lee $\mathrm{KB}$ Kim SR, et al. Thorax

2018;73:758-768.

\title{
Airway epithelial phosphoinositide 3-kinase- $\delta$ contributes to the modulation of fungi-induced innate immune response
}

\author{
Jae Seok Jeong, ${ }^{1}$ Kyung Bae Lee, ${ }^{1}$ So Ri Kim, ${ }^{1,2}$ Dong Im Kim, ${ }^{1}$ Hae Jin Park, ${ }^{1}$ \\ Hern-Ku Lee, ${ }^{3}$ Hyung Jin Kim, ${ }^{2,4}$ Seong Ho Cho, ${ }^{5}$ Narasaiah Kolliputi, ${ }^{5}$ Soon Ha Kim, \\ Yong Chul Lee 1,2 $^{1}$
}

\begin{abstract}
Background Respiratory fungal exposure is known to be associated with severe allergic lung inflammation. Airway epithelium is an essential controller of allergic inflammation. An innate immune recognition receptor, nucleotide-binding domain, leucine-richcontaining family, pyrin-domain-containing-3 (NLRP3) inflammasome, and phosphoinositide 3 kinase (PI3K)- $\delta$ in airway epithelium are involved in various inflammatory processes.
\end{abstract}

Objectives We investigated the role of NLRP3

inflammasome in fungi-induced allergic lung inflammation and examined the regulatory mechanism of NLRP3 inflammasome, focusing on PI3K- $\delta$ in airway epithelium.

Methods We used two in vivo models induced by exposure to Aspergillus fumigatus (Af) and Alternaria alternata $(A a)$, as well as an $A f$-exposed in vitro system. We also checked NLRP3 expression in lung tissues from patients with allergic bronchopulmonary aspergillosis (ABPA).

Results Assembly/activation of NLRP3 inflammasome was increased in the lung of $A f$-exposed mice. Elevation of NLRP3 inflammasome assembly/activation was observed in Af-stimulated murine and human epithelial cells. Similarly, pulmonary expression of NLRP3 in patients with ABPA was increased. Importantly, neutralisation of NLRP3 inflammasome derived IL-1 $\beta$ alleviated pathophysiological features of $A f$-induced allergic inflammation. Furthermore, PI3K- $\delta$ blockade improved $A f$-induced allergic inflammation through modulation of NLRP3 inflammasome, especially in epithelial cells. This modulatory role of PI3K- $\delta$ was mediated through the regulation of mitochondrial reactive oxygen species (mtROS) generation. NLRP3 inflammasome was also implicated in Aa-induced eosinophilic allergic inflammation, which was improved by PI3K- $\delta$ blockade.

Conclusion These findings demonstrate that fungiinduced assembly/activation of NLRP3 inflammasome in airway epithelium may be modulated by $\mathrm{PI} 3 \mathrm{~K}-\delta$, which is mediated partly through the regulation of mtROS generation. Inhibition of PI3K- $\delta$ may have potential for treating fungi-induced severe allergic lung inflammation.

\section{INTRODUCTION}

Bronchial asthma is recognised as a heterogeneous clinical syndrome, in which complex interplay

\section{Key messages}

What is the key question?

- Phosphoinositide 3-kinase (PI3K)- $\delta$ isoform has been shown as an important mediator of allergic lung inflammation, including the fungiinduced form; however, a role of PI3K- $\delta$ in the modulation of innate immune response against fungal allergens has not been reported.

What is the bottom line?

- PI3K- $\delta$ is critically implicated in fungi-induced allergic lung inflammation through regulation of nucleotide-binding domain, leucine-richcontaining family, pyrin-domain-containing-3 (NLRP3) inflammasome assembly/activation, which is mediated partly through the regulation of mitochondrial reactive oxygen species generation, particularly in the airway epithelium.

Why read on?

- Inhibition of PI3K- $\delta$ signalling may have potential for treating fungi-induced severe allergic lung inflammation in humans.

between innate and adaptive immune response is central to airway inflammation. ${ }^{1}$ Among many cells, airway epithelium which expresses various pattern recognition receptors (PRRs) ${ }^{2}$ is the first line of defence on encountering allergens and an essential controller of immune responses. ${ }^{3}$ Particularly, the nucleotide-binding domain, leucinerich repeat containing receptors (NLRs) represent one important PRR family in the intracellular compartment. ${ }^{4}$

Several members of NLRs are involved in the activation of caspase- 1 through assembling cytosolic multiprotein complexes called inflammasomes, leading to cleavage of the proinflammatory IL-1 family of cytokines, such as pro-IL-1 $\beta$ into its bioactive form, IL-1 $\beta$. Nucleotide-binding domain, leucine-rich-containing family, pyrin-domain-containing-3 (NLRP3) inflammasome consisting of NLRP3, apoptosis-associated speck-like protein containing a carboxy-terminal caspase-recruitment domain (ASC), and caspase- 1 is the most characterised inflammasome. ${ }^{5}$ NLRP3 inflammasome has 
been reported to be implicated in diverse human disorders. ${ }^{6-8}$ Assembly/activation of NLRP3 inflammasome is induced through several intermediate factors. ${ }^{5}$ Specifically, functional association between NLRP3 inflammasome and intracellular organelles, including endoplasmic reticulum (ER) and mitochondria, is a key regulatory mechanism of NLRP3 inflammasome activation, partly via release of mitochondrial reactive oxygen species (mtROS). ${ }^{9}{ }^{10}$ Recently, we showed that NLRP3 inflammasome activation by mtROS in airway epithelial cells (EpCs) is important in allergic lung inflammation. ${ }^{11}$

Fungal exposure has been recognised as a precipitating factor for development and exacerbation of asthma. ${ }^{12}$ According to a previous study, over $50 \%$ of patients with severe asthma were sensitised to one or more fungi. ${ }^{13}$ Among various species, Aspergillus fumigatus $(A f)$ and Alternaria alternata $(A a)$ are common airborne fungi implicated in severe asthma. ${ }^{12} 14$ Particularly, the impact of $A f$ on asthma severity has been well characterised ${ }^{15}$ and it is also involved in allergic bronchopulmonary aspergillosis (ABPA), a representative allergic bronchopulmonary mycosis which is assumed as an extreme spectrum of allergic response against fungi. ${ }^{12} 1416$ In patients with fungi-associated allergic lung inflammation, conventional treatment using inhaled corticosteroid (CS) seems to be inadequate and other treatment modalities such as systemic CS and antifungal agents have been suggested. ${ }^{17}$ Nevertheless, limited information exists on the mechanisms of how fungi are linked to the allergic inflammation.

Although NLRP3 inflammasome is vital in anti-fungal immune response, ${ }^{18}$ the role of NLRP3 inflammasome in fungus-induced allergic lung inflammation remains poorly understood. Contradictory findings exist regarding the involvement of NLRP3 inflammasome in allergic lung inflammation. ${ }^{811} 1920$ Given the involvement of NLRP3 inflammasome in many pathological conditions, controlling NLRP3 inflammasome may offer beneficial effects in fungi-induced allergic lung disorders. We previously demonstrated that activation of phosphoinositide 3-kinase $(\mathrm{PI} 3 \mathrm{~K})-\delta$ in lung EpCs contributes to allergic lung inflammation through the control of inflammatory mediators. ${ }^{21}$ In addition, PI3K- $\delta$ in airway EpCs was shown to be involved in fungi-induced allergic lung inflammation through modulating ER stress, ${ }^{22}$ which has been known as a regulator of NLRP3 inflammasome assembly/activation. ${ }^{1023}$

In this study, the role of NLRP3 inflammasome in fungi-induced allergic lung inflammation was evaluated using two murine models induced by $A f$ and $A a$. We also examined whether PI3K- $\delta$ influences NLRP3 inflammasome assembly/activation in fungi-induced allergic lung inflammation, especially in airway EpCs using Af-stimulated primary cultured EpCs. Lastly, we checked expression of NLRP3 in lung tissues from patients with ABPA to verify the involvement of NLRP3 inflammasome in fungi-induced human allergic lung disorder.

\section{METHODS}

Detailed methods are provided in the online supplement.

\section{Animals and experimental protocol}

Female C57BL/6 mice, 8-10 weeks of age, were used. All animal experiments were approved by the Institutional Animal Care and Use Committee of the Chonbuk National University and were performed in accordance with the ARRIVE (Animal Research: Reporting of In Vivo Experiments) guidelines. For the generation of fungi-induced allergic lung inflammation murine models, mice were exposed to $A f$ or $A a$ extract (Greer Laboratories, Lenoir, North Carolina, USA) as previously described. ${ }^{22} 24$

\section{Cell culture and treatment}

Primary cultured murine tracheal EpCs were isolated under sterile conditions and treated with IC87114, a selective inhibitor of $\mathrm{PI} 3 \mathrm{~K}-\delta$, or PI3K- $\delta$-specific siRNA as described previously. ${ }^{22}$ Normal human bronchial epithelial (NHBE) cells were purchased from Lonza (Walkersville, Maryland, USA).

\section{Immunofluorescence staining for NLRP3, caspase-1 and ASC and cytoplasmic localisation of mtROS}

Immunofluorescence staining and cytoplasmic localisation of mtROS were performed as previously described. ${ }^{22}$

\section{Western blot analysis}

Protein levels were determined as previously described. ${ }^{22}$

\section{Immunoprecipitation}

Co-immunoprecipitation (IP) of NLRP3 and interacting proteins was performed using Dynabeads protein G (ThermoFisher Scientific Inc., Waltham, Massachusetts, USA) according to the manufacturer's instructions.

\section{Trichloroacetic acid-mediated protein precipitation}

Trichloroacetic acid (TCA)-mediated protein precipitation of bronchoalveolar lavage (BAL) fluids was performed as described elsewhere. ${ }^{26}$

\section{ASC oligomerisation assay}

Cross-linking of ASC oligomers using disuccinimidyl suberate cross-linker was performed as previously described elsewhere. ${ }^{27}$

\section{Determination of airway responsiveness to methacholine}

Invasive measurement was performed as previously described. ${ }^{22}$

\section{Statistics}

We used SPSS software (version 18.0, SPSS, Chicago, Illinois, USA). Data are expressed as mean \pm SEM. Statistical comparisons were performed using one-way ANOVA followed by the Scheffe's test. Significant differences between two groups (analyses between control and $A f$-exposed groups and analyses between $A f$-exposed wild type and $A f$-exposed p110 knockout (KO) mice) were determined using unpaired t test. A value of $P<0.05$ was considered statistically significant.

\section{RESULTS \\ NLRP3 inflammasome is implicated in Af-induced allergic lung inflammation}

First, we determined expression of NLRP3 inflammasome components and IL-1 $\beta$ in Af-sensitised/challenged mice. Immunofluorescence intensities of NLRP3 and caspase-1 in BAL cells were remarkably increased in $A f$-exposed mice (figure $1 \mathrm{~A}$ and online supplementary figure S1A, B). We observed the co-localisation of NLRP3 and caspase-1, predominantly in the cytoplasmic area (figure $1 \mathrm{~A}$ and online supplementary figure S1C). Immunofluorescence intensities of ASC and NLRP3 and their co-localisation were also increased, especially in cytoplasm, in BAL cells (figure $1 \mathrm{~B}$ and online supplementary figure S1D-F). Furthermore, IL-1 $\beta$ was elevated in BAL fluids (figure 1C). Similarly, immunofluorescence intensities of NLRP3 and caspase-1 and their co-localisation were increased, particularly in epithelial cell layers, in lung tissues (figure 1D and online supplementary figure S1G-I). IL-1 $\beta$ was also increased in lung tissues (figure $1 \mathrm{E}$ ). 


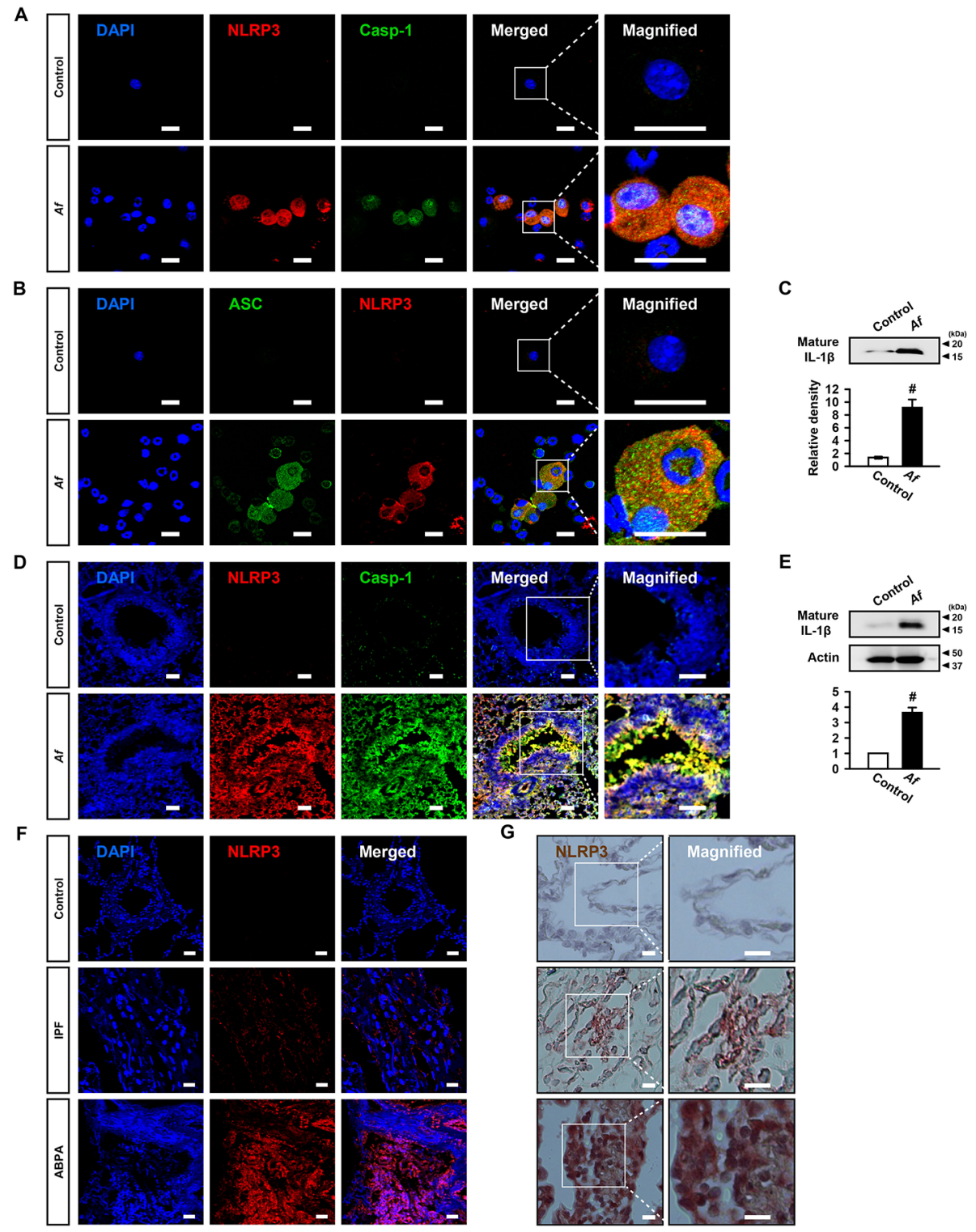

Figure 1 Nucleotide-binding domain, leucine-rich-containing family, pyrin-domain-containing-3 (NLRP3) inflammasome is involved in Aspergillus fumigatus ( $A f$ )-induced allergic lung inflammation. ( $A$ and $B$ ) Representative confocal images of bronchoalveolar lavage (BAL) cells show the localisation of NLRP3 (red), caspase-1 (Casp-1, green) and apoptosis-associated speck-like protein containing a carboxy-terminal caspase-recruitment domain (ASC; green) from saline-exposed (control) or Af-exposed (Af) mice. 4',6-Diamidino-2-phenylindole (DAPI) stain was used for nuclear localisation. Bars indicate $20 \mu \mathrm{m}$. (C) Representative immunoblots and densitometric analyses of IL-1 $\beta$ in BAL fluids from saline-exposed (control) and $A f$-exposed (Af) mice. Bars represent mean \pm SEM from six mice per group. ${ }^{\sharp} P<0.05$ versus control. (D) Representative confocal images show the localisation of NLRP3 (red) and Casp-1 (green) in lung tissues from saline-exposed (control) and Af-exposed (Af) mice. Bars indicate $50 \mu$ m. (E) Representative immunoblots and densitometric analyses of IL-1 $\beta$ in lung tissues from saline-exposed (control) and $A f$-exposed ( $A f$ ) mice. Bars represent mean \pm SEM from six mice per group. ${ }^{\#} P<0.05$ versus control. (F) Representative confocal image for NLRP3 in lung tissues from a healthy control, a patient with idiopathic pulmonary fibrosis (IPF; disease control), and a patient with allergic bronchopulmonary aspergillosis (ABPA), respectively. Bars indicate $50 \mu \mathrm{m}$. (G) Representative immunohistochemical staining of NLRP3 in lung tissues of a healthy person, a patient with IPF and a patient with ABPA. Brown-stained cells were considered to express the NLRP3 protein. Bars indicate $20 \mu \mathrm{m}$.

\section{NLRP3 is increased in lung tissues from patients with ABPA}

Aspergillus-related disorders encompass a spectrum of diseases. ${ }^{28}$ Notably, ABPA represents the most severe type of T helper type 2 (Th2)-mediated allergic response against aspergillus species. ${ }^{29} \mathrm{We}$ evaluated immunofluorescence and immunohistochemical analyses for NLRP3 in lung tissues from patients with ABPA, patients with idiopathic pulmonary fibrosis (IPF), and healthy controls, because NLRP3 expression is a limiting factor in NLRP3 inflammasome activation. ${ }^{30}$ Immunofluorescence intensity of NLRP3 was increased in patients with ABPA or IPF. However, increase of NLRP3 in patients with ABPA was significantly higher than in patients with IPF (figure $1 \mathrm{~F}$ and online supplementary figure S1J). Immunohistochemical analyses also showed that immunoreactive NLRP3 was increased in patients with ABPA or IPF. However, immunoreactive NLRP3 was significantly increased in patients with ABPA (figure $1 G$ and online supplementary figure $\mathrm{S} 1 \mathrm{~K})$. These data indicate that $A f$ exposure may be related to the NLRP3 inflammasome activation in human lung disorder, though we cannot rule out the possibility of the involvement of other factors. 
Af leads to NLRP3 inflammasome activation in tracheal EpCs

Airway epithelium is the first contact site with Af and a key modulator of innate immune response against $A f{ }^{31}$ We checked expression of NLRP3 inflammasome components in Af-stimulated tracheal EpCs. Immunofluorescence intensities of NLRP3 and caspase- 1 were increased after $A f$ stimulation (figure $2 \mathrm{~A}$ and online supplementary figure S2A-C). Moreover, cytoplasmic co-localisation of NLRP3 and caspase-1 was increased (figure 2A and online supplementary figure S2A, D). Similarly, immunofluorescence intensities of ASC and NLRP3 and their cytoplasmic co-localisation were increased in these cells (figure $2 \mathrm{~B}$ and online supplementary figure S2E-H).

\section{IL-1 $\beta$ inhibition improves $A f$-induced allergic lung inflammation}

To investigate whether NLRP3 inflammasome-derived IL-1 $\beta$ is implicated in $A f$-induced allergic lung inflammation, we evaluated the effects of IL-1 $\beta$ blockade on features of the disease. First, we determined the effect of MCC 950, a selective inhibitor of NLRP3 inflammasome, ${ }^{32}$ on IL-1 $\beta$ in BAL fluids. Results showed that $A f$-induced increase of IL- $1 \beta$ was significantly lowered by MCC 950 (figure 2C), indicating that NLRP3 inflammasome plays a key role in IL-1 $\beta$ generation in Af-exposed mice. Notably, IL-1 $\beta$ neutralising antibody $(\mathrm{Ab})$ treatment improved respiratory system resistance $\left(\mathrm{R}_{\mathrm{rs}}\right)$ (figure $2 \mathrm{D}$ ). Increased numbers of total cells, lymphocytes, and eosinophils in BAL fluids were lowered by IL- $1 \beta$ neutralising $\mathrm{Ab}$ (figure $2 \mathrm{E}$ ). Furthermore, $A$-exposed mice administered IL-1 $\beta$ neutralising $A b$ showed reduction in the infiltrations of inflammatory cells into the peribronchiolar and perivascular regions (figure $2 \mathrm{~F}-\mathrm{H}$ ).

\section{PI3K- $\delta$ blockade ameliorates $A f$-induced allergic lung inflammation through regulation of NLRP3 inflammasome assembly/activation}

First, through assessing the therapeutic effects of IC87114, a selective inhibitor of $\mathrm{PI} 3 \mathrm{~K}-\delta$, in $A f$-sensitised/challenged mice, we observed that PI $3 \mathrm{~K}-\delta$ activity contributes to overall activities of class I PI3Ks in the lung and plays a key role in $A f$-induced allergic lung inflammation (online supplementary figure S3). Then, we determined the effect of PI3K- $\delta$ blockade on NLRP3 inflammasome assembly/activation in vivo. Af-induced increases in immunofluorescence intensities of NLRP3, caspase-1, and ASC and their cytoplasmic co-localisations in BAL cells were notably reduced by IC 87114 (figure 3A, B and online supplementary figure S4). Viability assay of BAL cells after intratracheal administration of IC87114 revealed that there was no significant toxic effect of the inhibitor on cells (online supplementary figure S5). IP assay also showed that $A f$-induced increase of NLRP3 co-precipitated with caspase-1 or ASC in lung tissues was reduced by IC87114 (figure 3C). Furthermore, Af-induced increases of NLRP3 and caspase-1 p10 (an autoprocessed cleaved form of caspase-1) in lung tissues were significantly reduced by IC87114 (figure 3D, E). Increased levels of IL-1 $\beta$ in lung tissues and BAL fluids were also lowered by IC87114 (figure 3F-H). To further determine whether PI3K- $\delta$ affects NLRP3 inflammasome, KO mice without the catalytic subunit of PI3K- $\delta(\mathrm{p} 110 \delta)$ were used. Whereas $A f$ exposure led to the increase of $\mathrm{p} 110 \delta$ in wild type (WT) mice, p110 $\delta$ expression was absent in lung tissues of $A f$-exposed $\mathrm{p} 110 \delta \mathrm{KO}$ mice. In addition, the extent of the infiltration of numerous inflammatory cells into the peribronchial and perivascular regions was much lower in $A f$-exposed $\mathrm{p} 110 \delta$

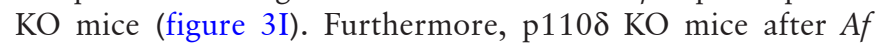

exposure demonstrated the lower levels of NLRP3 and cleaved caspase-1 in lung tissues (figure $3 \mathrm{~J}, \mathrm{~K}$ ). In addition, $A$-exposed $\mathrm{p} 110 \delta \mathrm{KO}$ mice had lower levels of IL-1 $\beta$ in lung tissues and BAL fluids (figure $3 \mathrm{~L}, \mathrm{M}$ ).

\section{$A f$-induced assembly/activation of NLRP3 inflammasome in bronchial EpCs is regulated by $\mathrm{PI} 3 \mathrm{~K}-\delta$}

Then, we examined whether PI3K- $\delta$ modulates NLRP3 inflammasome in airway EpCs. IC87114 substantially limited $A f$-induced increases in immunofluorescence intensities of NLRP3, caspase-1 and ASC and their cytoplasmic co-localisations in EpCs (figure 4A, B and online supplementary figure S6A-F). We also examined the formation of ASC oligomerisation, a key process in NLRP3 inflammasome assembly/activation, ${ }^{32}$ in these cells and IC87114 substantially lowered $A f$-induced formation of ASC monomers and high order complexes (figure 4C). Furthermore, when PI3K- $\delta$ in EpCs was knocked down, $A f$-induced expression of NLRP3 and caspase-1 and their cytoplasmic co-localisation were substantially lowered (figure 4D and online supplementary figure S6G-I). Supporting these data, $A f$-induced increases of NLRP3 and IL-1 $\beta$ in cell lysates were reduced by IC 87114 or PI3K- $\delta$ siRNA (online supplementary figure S6J-M). Meanwhile, ROS generation acts as one of the major upstream signals for NLRP3 inflammasome activation. ${ }^{9}$ Mitochondrion is a major source of intracellular ROS, thereby being involved in NLRP3 inflammasome activation. ${ }^{9} 33$ To investigate the role of mtROS in $A f$-induced allergic lung inflammation, we determined the effects of a potent mtROS scavenger, NecroX- $5^{22}$, in $A f$-exposed mice. Fluorescence intensity of mtROS was increased in cytoplasm of BAL cells, mainly macrophages and eosinophils, from $A$-exposed mice (figure 4E, F and online supplementary figure $S 6 \mathrm{~N}$ ). NecroX-5 reduced $A f$-induced increase in fluorescence intensity of mtROS. Notably, NecroX-5 dramatically reduced the $A f$-induced increase of IL-1 $\beta$ (figure 4G). Moreover, NecroX-5 ameliorated $A$-induced allergic lung inflammation and airway hyper-responsiveness (AHR) (figure 4H-L). Furthermore, $A f$-induced mtROS generation was decreased by IC87114 in both BAL cells and EpCs (figure 4M, N and online supplementary figure $\mathrm{S} 6 \mathrm{O}, \mathrm{P}$ ).

\section{Af-stimulated NHBE cells replicate the in vitro murine studies}

To investigate whether PI3K- $\delta$ also modulates NLRP3 inflammasome in human epithelial cells, we checked expression of NLRP3 inflammasome components and their co-localisations in Af-stimulated NHBE cells. IC87114 significantly lowered the $A f$-induced increases in immunofluorescence intensities of NLRP3, caspase-1 and ASC and their cytoplasmic co-localisations in $A f$-stimulated NHBE cells (figure $5 \mathrm{~A}, \mathrm{~B}$ and online supplementary figure S7A-F). Furthermore, IC87114 lowered the $A f$-induced ASC-complex formation (figure $5 \mathrm{C}$ ). In the same vein, increased levels of NLRP3 and IL-1 $\beta$ in Af-stimulated NHBE cells were reduced by IC87114 (figure 5D, E). Similarly, when PI3K- $\delta$ was knocked down, increased levels of NLRP3 and IL-1 $\beta$ in Af-stimulated NHBE cells were significantly reduced (figure $5 \mathrm{~F}, \mathrm{G}$ ). Consistent with these observations, $A f$-induced increased immunofluorescence intensities of NLRP3 and caspase-1 and their cytoplasmic co-localisation were substantially lowered by PI $3 \mathrm{~K}-\delta$ siRNA (figure $5 \mathrm{H}$ and online supplementary figure S7G-I). These data suggest that PI3K- $\delta$ also regulates $A f$-induced assembly/activation of NLRP3 inflammasome in NHBE cells. Additionally, $A f$-induced 
A
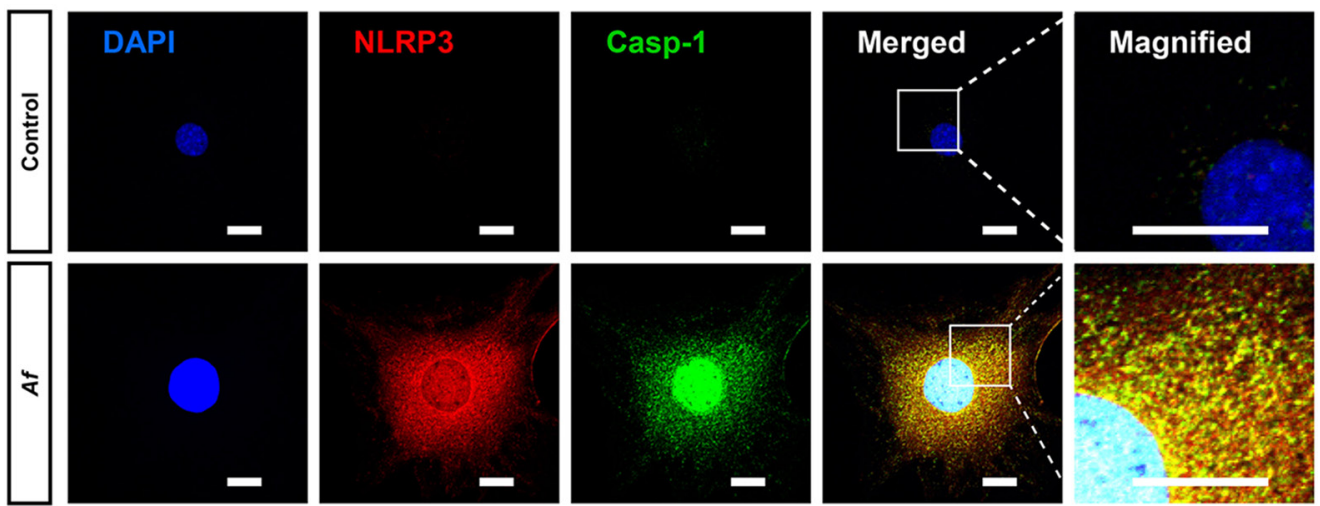

B
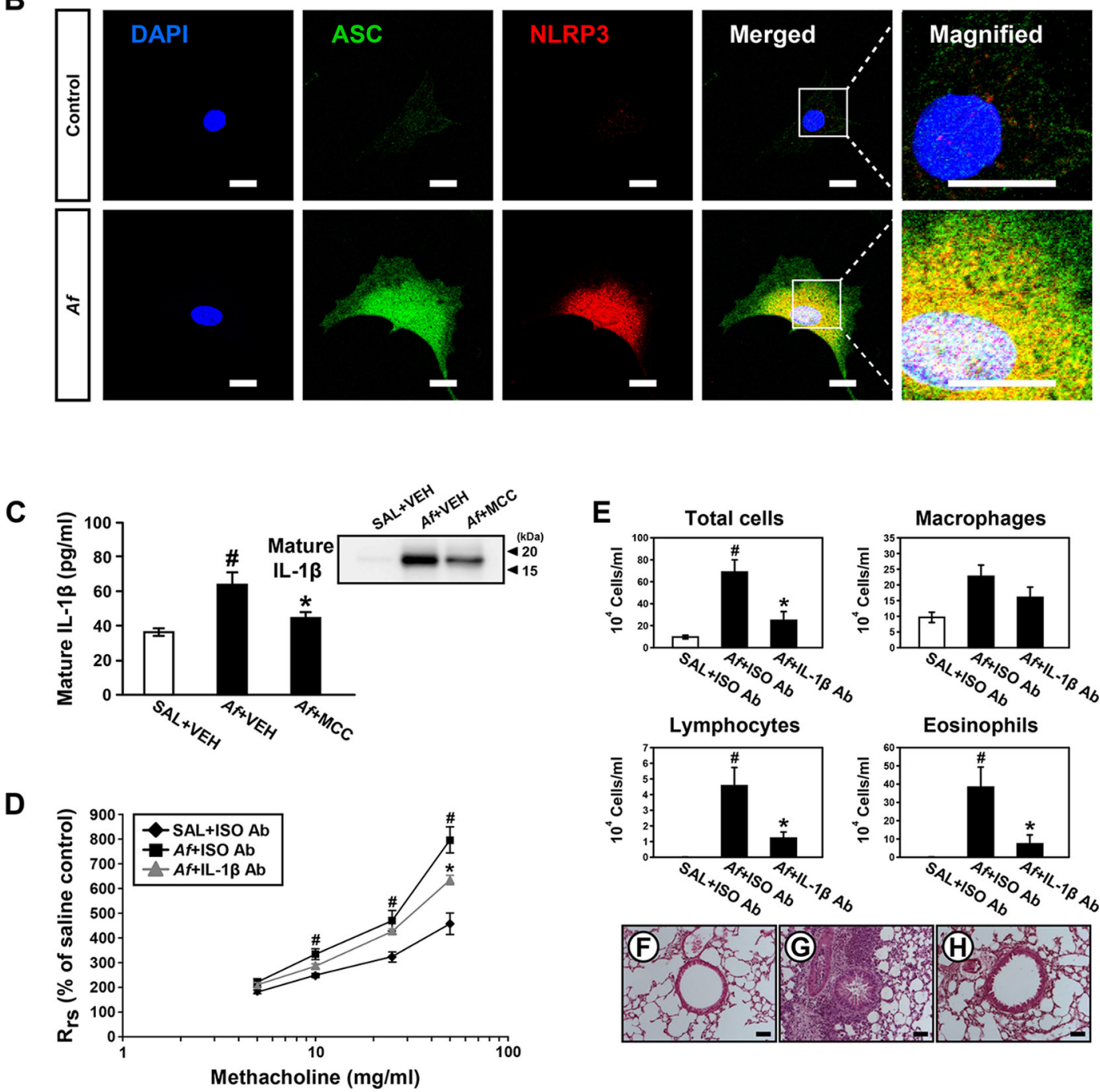

Figure 2 Stimulation with Aspergillus fumigatus ( $A f)$ leads to nucleotide-binding domain, leucine-rich-containing family, pyrin-domain-containing-3 (NLRP3) inflammasome assembly in tracheal epithelial cells (EpCs) and neutralisation of IL-1 $\beta$ improves $A f$-induced allergic lung inflammation. (A and B) Representative confocal images of tracheal EpCs show the localisation of NLRP3 (red), caspase-1 (Casp-1, green) and apoptosis-associated specklike protein containing a carboxy-terminal caspase-recruitment domain (ASC; green) in normal (control) and Af-stimulated (Af) cells. 4',6-Diamidino2-phenylindole (DAPI) stain was used for nuclear localisation. Bars indicate $20 \mu \mathrm{m}$. (C) Enzyme immunoassays of IL-1 $\beta$ in bronchoalveolar lavage (BAL) fluids from saline-exposed mice administered drug vehicle (SAL+VEH), $A f$-exposed mice administered drug vehicle $(A f+V E H)$, or $A f$-exposed mice administered MCC 950 ( $A f+\mathrm{MCC})$. Bars represent mean \pm SEM from six mice per group. ${ }^{\#} P<0.05$ versus $S A L+V E H ;{ }^{*} P<0.05$ versus $A f+V E H$. Right upper inset image demonstrates the representative immunoblot of IL-1 $\beta$ in BAL fluids. (D) Airway responsiveness assessed by invasive measurements in saline-exposed mice administered isotype antibody (Ab) (SAL+ISOAb), $A f$-exposed mice administered isotype $A b(A f+I S O A b)$, or $A f$-exposed mice administered anti-IL-1 $\beta$ neutralising $A b(A f+I L-1 \beta A b)$. Bars represent mean \pm SEM from six mice per group. ${ }^{\#} P<0.05$ versus $S A L+I S O A b ;{ }^{*} P<0.05$ versus $A f+I S O A b$. (E) Cellular changes in BAL fluids. Bars represent mean \pm SEM from five mice per group. ${ }^{\#} P<0.05$ versus $S A L+I S O A b ;{ }^{*} P<0.05$ versus $A f+I S O A b$. (F-H) Representative H\&E stained sections of the lung from SAL+ISOAb (F), $A f+I S O A b(G)$, and $A f+I L-1 \beta A b(H)$. Bars indicate $50 \mu m$. 
A

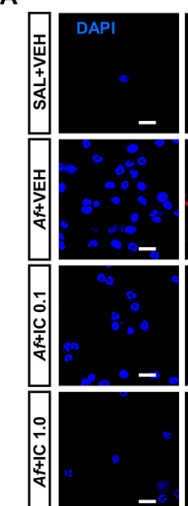

B
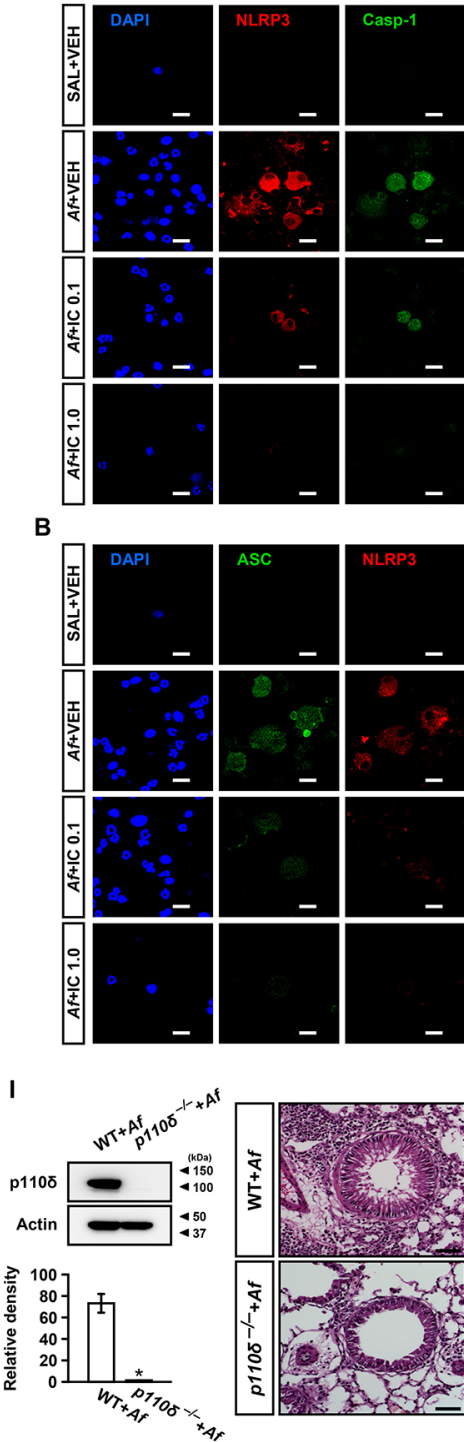
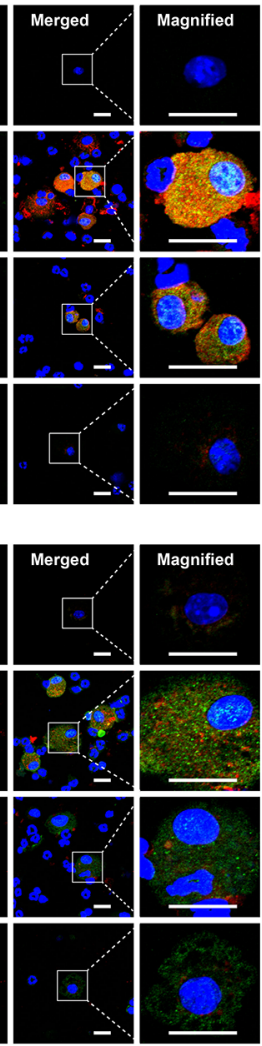

$\mathbf{J}$

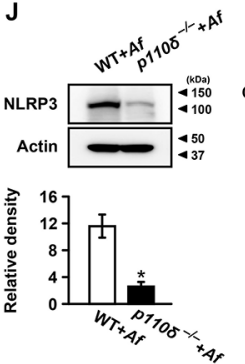

C

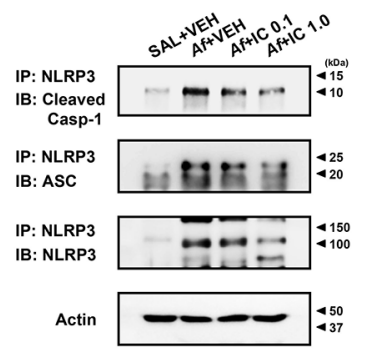

E
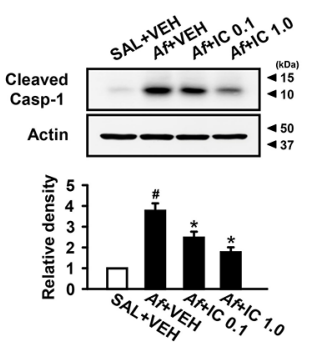

G

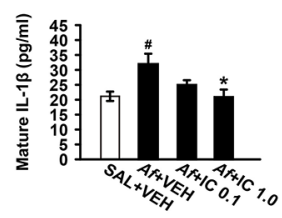

D

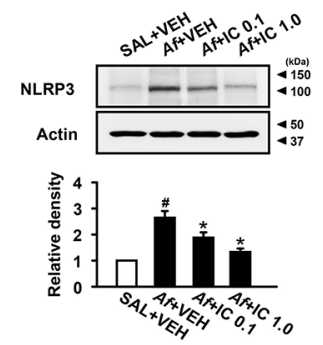

$\mathbf{F}$
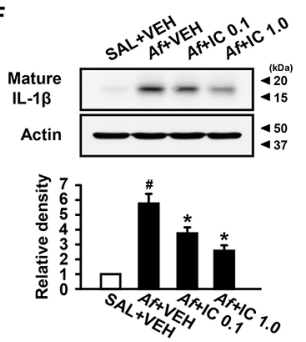

H
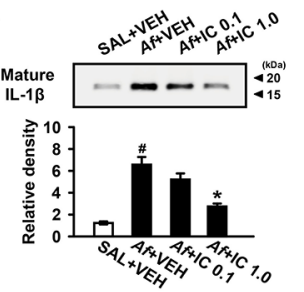

M
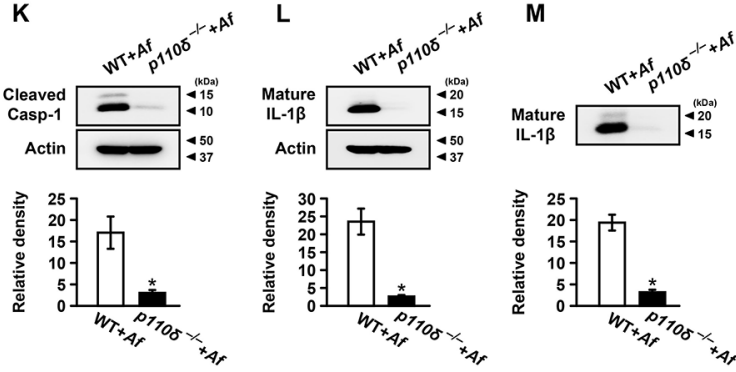

Figure 3 Inhibition of phosphoinositide 3-kinase (PI3K)- $\delta$ improves Aspergillus fumigatus (Af)-induced allergic lung inflammation through regulation of nucleotide-binding domain, leucine-rich-containing family, pyrin-domain-containing-3 (NLRP3) inflammasome assembly/activation. (A and B) Representative confocal images of bronchoalveolar lavage (BAL) cells show the localisation of NLRP3 (red), caspase-1 (Casp-1; green) and apoptosis-associated speck-like protein containing a carboxy-terminal caspase-recruitment domain (ASC; green) from saline-exposed mice administered drug vehicle (SAL+VEH), $A f$-exposed mice administered drug vehicle (Af+VEH), $A f$-exposed mice administered $0.1 \mathrm{mg} / \mathrm{kg} \mathrm{IC} 87114(A f+\mathrm{IC}$ 0.1 ) or $A f$-exposed mice administered $1.0 \mathrm{mg} / \mathrm{kg}$ IC87114 (Af+IC 1.0). 4',6-Diamidino-2-phenylindole (DAPI) stain was used for nuclear localisation. Bars indicate $20 \mu \mathrm{m}$. (C) Representative immunoblots of Casp-1 or ASC co-precipitated (IP) with NLRP3 in lung tissues. (D-F) Representative immunoblots and densitometric analyses of NLRP3 (D), Casp-1 (E) and IL-1 $\beta$ (F) in lung tissues. (G and H) Enzyme immunoassay (G) and representative immunoblots and densitometric analyses $(\mathrm{H})$ of IL-1 $\beta$ in BAL fluids. Bars represent mean \pm SEM from six mice per group. ${ }^{\#} P<0.05$ versus $\mathrm{SAL}+\mathrm{VEH} ;{ }^{*} P<0.05$ versus $A f+\mathrm{VEH}$. (I) Representative immunoblots and densitometric analyses of $\mathrm{p} 110 \delta$ in lung tissues and representative $\mathrm{H} \& \mathrm{E}$ stained sections of the lung from $A f$-exposed mice with p110 $\mathrm{KO}\left(\mathrm{p} 110 \delta^{-1-}\right)$ or WT. Bars indicate $50 \mu \mathrm{m}$. (J-M) Representative immunoblots and densitometric analyses of NLRP3 (J), Casp-1 (K) and IL-1 $\beta$ (L) in lung tissues, and IL-1 $\beta$ in BAL fluids (M) from $A f$-exposed mice with $p 110 \delta^{-1-}$ or WT. Bars represent mean \pm SEM from seven mice per group. ${ }^{\#} P<0.05$ versus $\mathrm{WT}+A f$.

generation of mtROS were significantly lowered by IC 87114 in NHBE cells (figure 5I and online supplementary figure S7J).

\section{PI3K- $\delta$ regulates NLRP3 inflammasome in Aa-induced allergic lung inflammation}

To clarify the involvement of NLRP3 inflammasome in fungi-induced allergic lung inflammation, we evaluated the effects of MCC 950 on allergic lung inflammation induced by $A a$. Treatment with MCC 950 significantly reduced $A a$-induced increases of eosinophil-dominant inflammatory cells infiltration, Th2 cytokines and IL-1 $\beta$ in the lung of mice (online supplementary figure S8). We then explored whether PI3K- $\delta$ contributes to $A a$-induced allergic lung inflammation, and if PI3K- $\delta$ regulates NLRP3 inflammasome activation. Data showed that $A a$-induced increases of airway inflammatory cells infiltration and pulmonary Th2 cytokines were significantly reduced by treatment with IC87114. Interestingly, $A a$-induced increases of IL-1 $\beta$ in lung tissues and BAL fluids were significantly lowered by IC87114 (online supplementary figure S9). 
A

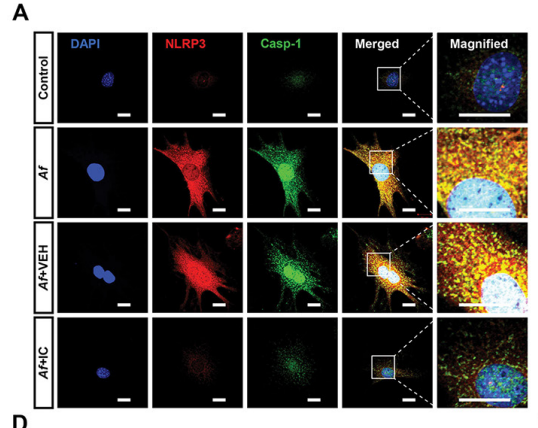

D

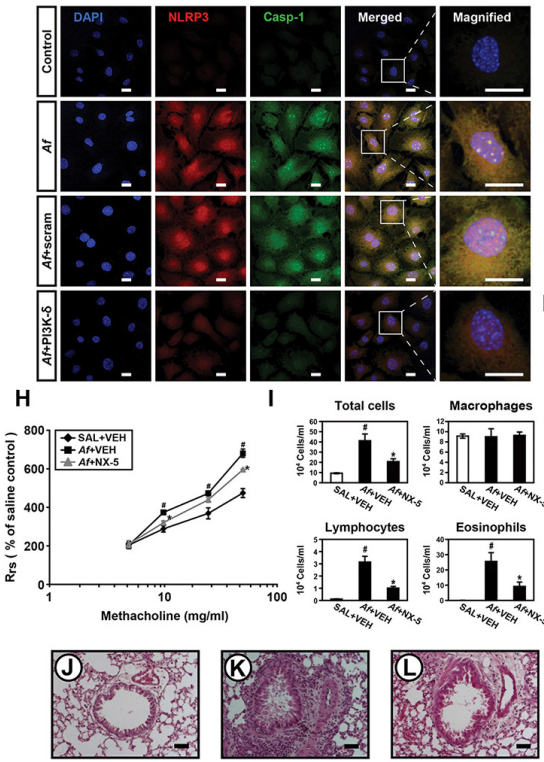

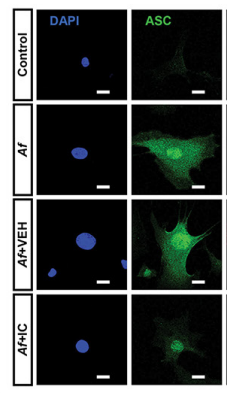

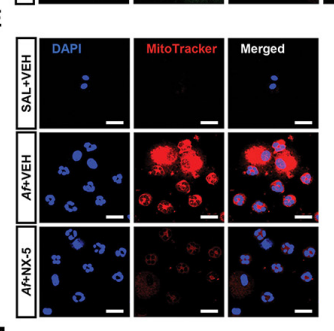

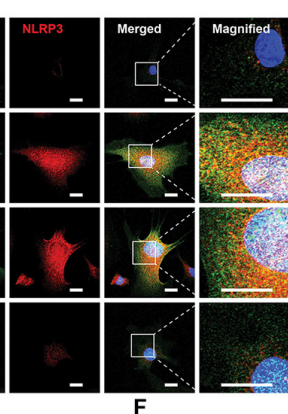

$\mathrm{F}$

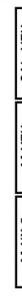

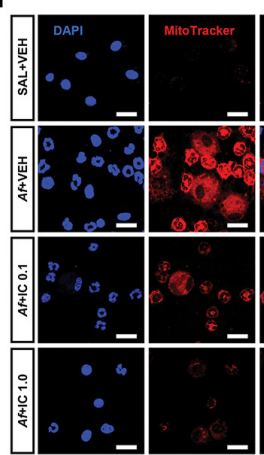
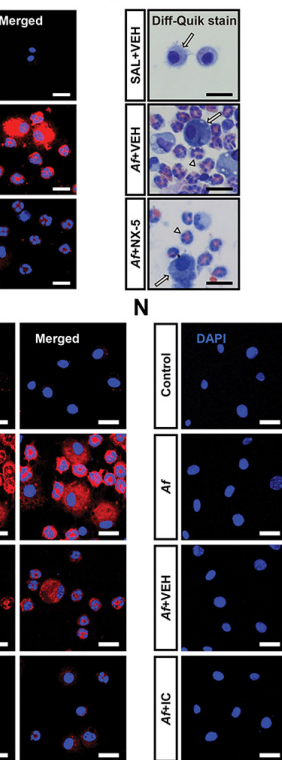

C

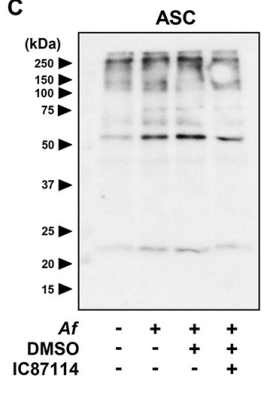

G
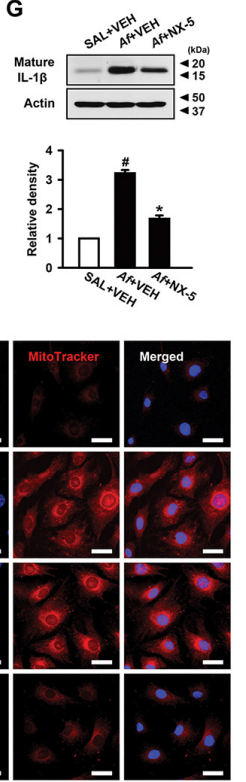

Figure 4 Aspergillus fumigatus (Af)-induced nucleotide-binding domain, leucine-rich-containing family, pyrin-domain-containing-3 (NLRP3) inflammasome assembly/activation is regulated by phosphoinositide 3-kinase (PI3K)- $\delta$ in epithelial cells (EpCs). (A and B) Representative confocal images of tracheal EpCs show the localisation of NLRP3 (red), caspase-1 (Casp-1; green) and apoptosis-associated speck-like protein containing a carboxy-terminal caspase-recruitment domain (ASC; green) in the control (no treatment), Af-stimulated cells (Af), Af-stimulated cells administered drug vehicle $(A f+V E H)$, and $A f$-stimulated cells administered IC87114 ( $A f+I C)$. 4',6-Diamidino-2-phenylindole (DAPI) stain was used for nuclear localisation. Bars indicate $20 \mu \mathrm{m}$. (C) Representative immunoblot of ASC monomers and high order complexes in cross-linked cytosolic pellets from Af-stimulated EpCs in the presence and absence of IC87114. (D) Representative confocal images of tracheal EpCs show the localisation of NLRP3 (red) and Casp-1 (green) in the control (no treatment), $A f$-stimulated cells ( $A f), A f$-stimulated cells administered scrambled siRNA ( $A f+S c r a m)$, or $A f$-stimulated cells administered PI3K- $\delta$ siRNA (Af+PI3K- $\delta$ ). Bars indicate $20 \mu \mathrm{m}$. (E) Representative confocal images show the localisation of mitochondrial ROS (mtROS) in bronchoalveolar lavage (BAL) cells from saline-exposed mice administered drug vehicle (SAL+VEH), $A f$-exposed mice administered drug vehicle $(A f+V E H)$, or $A f$-exposed mice administered NecroX-5 (Af+NX-5). Bars indicate $20 \mu m$. (F) Cellular changes in BAL fluids of Af-exposed mice. Arrows and arrowheads indicate macrophages and eosinophils, respectively. Bars indicate $20 \mu \mathrm{m}$. (G) Representative immunoblots and densitometric analyses of IL- $1 \beta$ in lung tissues. Bars represent mean \pm SEM from seven mice per group. ${ }^{\#} \mathrm{P}<0.05$ versus $\mathrm{SAL}+\mathrm{VEH}$; ${ }^{*} P<0.05$ versus $A f+V E H$. (H) Airway responsiveness assessed by invasive measurements from $S A L+V E H, A f+V E H$ or $A f+N X-5$. Bars represent mean $\pm S E M$ from six mice per group. (I) Cellular changes in bronchoalveolar lavage fluids. Bars represent mean $\pm \mathrm{SEM}$ from six mice per group. ${ }^{\#} \mathrm{P}<0.05$ versus $\mathrm{SAL}+\mathrm{VEH}$; ${ }^{*} P$ $<0.05$ versus $A f+V E H$. (J-L) Representative H\&E stained sections of the lung from SAL+VEH $(J), A f+V E H(K)$ and $A f+N X-5(L)$. Bars indicate $50 \mu m$. (M) Representative confocal images show the localisation of mtROS in BAL cells from saline-exposed mice administered drug vehicle $(S A L+V E H)$, $A f-$ exposed mice administered drug vehicle ( $A f+\mathrm{VEH})$, $A f$-exposed mice administered $0.1 \mathrm{mg} / \mathrm{kg} \mathrm{IC} 87114(A f+\mathrm{IC} 0.1)$ or $A f$-exposed mice administered $1.0 \mathrm{mg} / \mathrm{kg} \mathrm{IC87114}(A f+\mathrm{IC}$ 1.0). Bars indicate $20 \mu \mathrm{m}$. (N) Representative confocal images of tracheal EpCs show the localisation of mtROS in the control, $A f, A f+\mathrm{VEH}$ and $A f+\mathrm{IC}$. Bars indicate $10 \mu \mathrm{m}$.

\section{CS-resistant NLRP3 inflammasome contributes to Af-induced allergic lung inflammation}

CS resistance is a common feature in severe allergic lung inflammation. ${ }^{34}$ Recently, we showed that the current $A f$-induced model represents a CS-resistant severe phenotype. ${ }^{22}$ Therefore, we examined whether or not CS can influence NLRP3 inflammasome activation in the present two murine models. Data showed that dexamethasone did not improve the Af-induced increases of AHR, eosinophil-dominant inflammatory cells infiltration, and Th2 cytokines in the lung of mice (online supplementary figure S10A-I). In contrast, dexamethasone substantially improved the $A a$-induced increases of AHR, eosinophil-dominant inflammatory cell infiltration, and Th2 cytokines in the lung (online supplementary figure S10J-Q). Interestingly, increases of IL-1 $\beta$ in lung tissues and BAL fluids from Af-exposed mice were not lowered by dexamethasone. In contrast, increases of IL-1 $\beta$ in lung tissues and BAL fluids from $A a$-exposed mice were significantly reduced by dexamethasone (online supplementary figure S11A-D). Similarly, dexamethasone treatment failed to lower the $A f$-induced increases of NLRP3 and caspase-1 in lung tissues. However, $A a$-induced increases of 
A

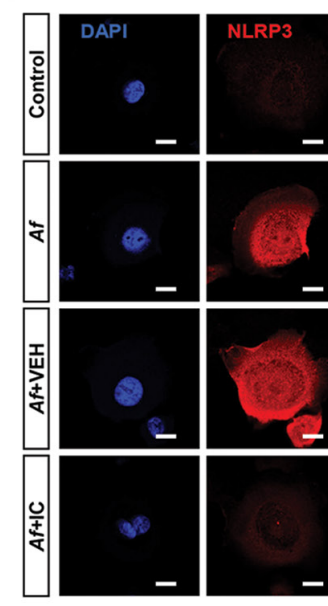

C

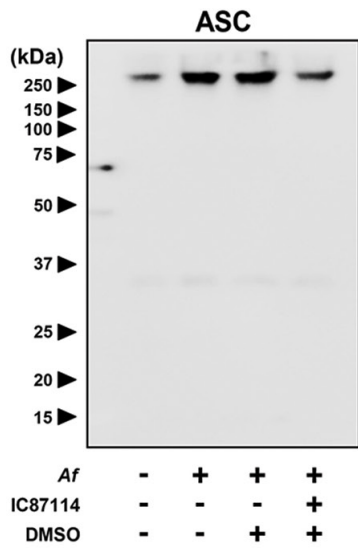

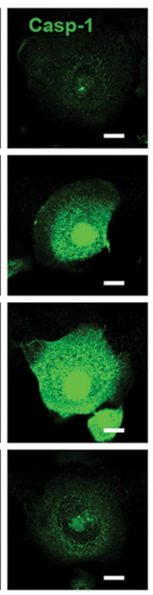

D
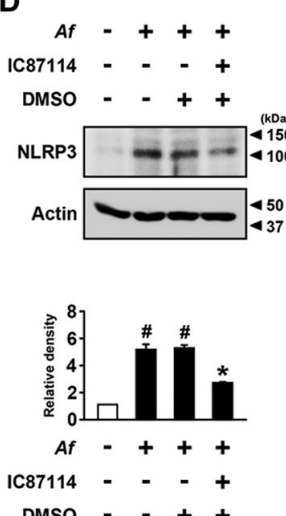
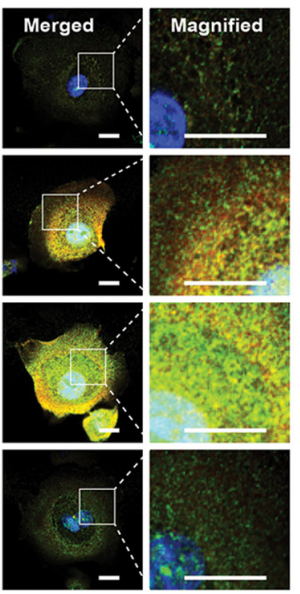

B

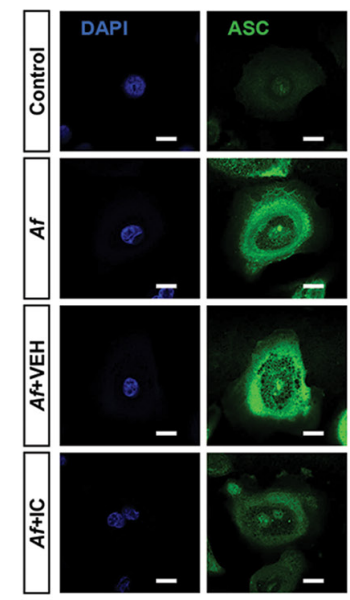

F

E
Af -+++

NT SiRNA - - +

PI3K- $\delta$ SiRNA - - - +

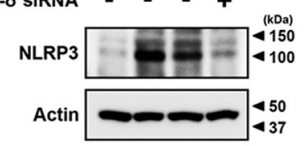

Actin $\longrightarrow=0$

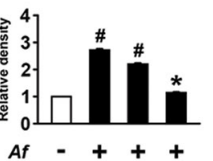

NT SiRNA - - +

PI3K- $\delta$ SIRNA - - +

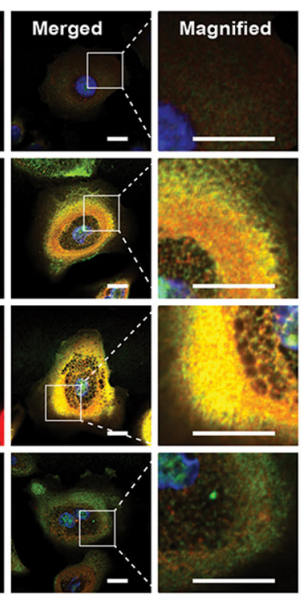

G
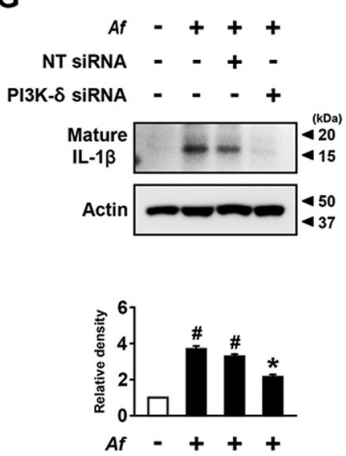

NT SiRNA - - +

PI3K- $\delta$ SIRNA - - - +
H

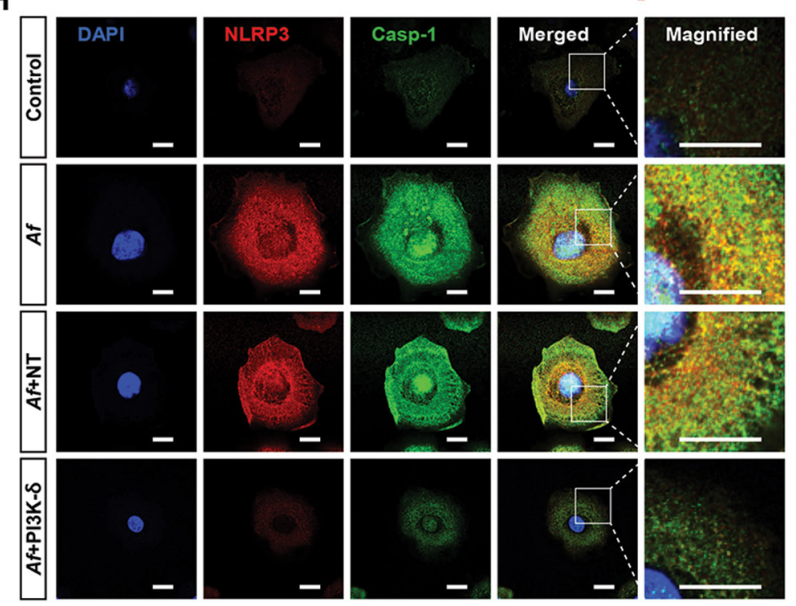

I

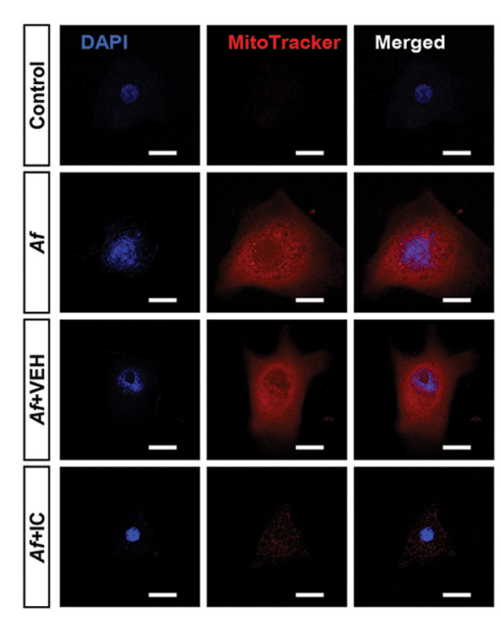

Figure 5 Phosphoinositide 3-kinase (PI3K)- $\delta$ regulates Aspergillus fumigatus (Af)-induced nucleotide-binding domain, leucine-rich-containing family, pyrin-domain-containing-3 (NLRP3) inflammasome assembly/activation in normal human bronchial epithelial (NHBE) cells. (A and B) Representative confocal images of NHBE cells show the localisation of NLRP3 (red), caspase-1 (Casp-1; green) and apoptosis-associated speck-like protein containing a carboxy-terminal caspase-recruitment domain (ASC; green) in the control (no treatment), $A f$-stimulated cells (Af), $A f$-stimulated cells administered drug vehicle ( $A f+\mathrm{VEH})$, or $A f$-stimulated cells administered IC87114 (Af+IC). 4',6-Diamidino-2-phenylindole (DAPI) stain was used for nuclear localisation. Bars indicate $20 \mu \mathrm{m}$. (C) Representative immunoblot of ASC monomers and high order complexes in cross-linked cytosolic pellets from Af-stimulated NHBE cells in the presence and absence of IC87114. (D and E) Representative immunoblots and densitometric analyses of NLRP3 (D) and IL-1 $\beta$ (E) after stimulation with Af in NHBE cells in the presence or absence of IC87114. Bars represent mean \pm SEM from three independent experiments. ${ }^{\#} P<0.05$ versus control; ${ }^{*} P<0.05$ versus cells stimulated with $A f$ alone. (F and $\mathrm{G}$ ) Representative immunoblots and densitometric analyses of NLRP3 (F) and IL-1 $\beta(G)$ after stimulation with $A f$ in NHBE cells transfected with either non-targeting siRNA (NT siRNA) or PI3K- $\delta$ siRNA. Bars represent mean \pm SEM from three independent experiments. ${ }^{\#} P<0.05$ versus control; * $P<0.05$ versus cells stimulated with $A f$ transfected with NT siRNA. (H) Representative confocal images of NHBE cells show the localisation of NLRP3 (red) and Casp-1 (green) in the control (no treatment), $A f$-stimulated cells $(A f), A f$-stimulated cells administered NT siRNA $(A f+N T)$, or $A f$-stimulated cells administered PI3K- $\delta$ siRNA $(A f+\mathrm{PI} 3 \mathrm{~K}-\delta)$. Bars indicate $20 \mu \mathrm{m}$. (I) Representative confocal images of NHBE cells show the localisation of mitochondrial reactive oxygen species (mtROS) in the control, $A f, A f+\mathrm{VEH}$ or $A f+\mathrm{IC}$. Bars indicate $20 \mu \mathrm{m}$. 
NLRP3 and caspase- 1 were significantly reduced by dexamethasone (online supplementary figure S11E-H).

\section{Effects of dexamethasone on mtROS generation and nuclear translocation of nuclear factor- $\mathrm{B}$ in lungs of two different murine models}

Oxidative stress is closely involved in the development of CS resistance in intractable pulmonary disorders, including severe asthma and chronic obstructive pulmonary disease. ${ }^{34}$ In addition, generation of mtROS is implicated in CS-resistant inflammation in the lung. ${ }^{22}$ To investigate the molecular mechanisms which underlie the different responses to CS between the two mouse models, we determined mtROS and the related activation of nuclear factor (NF)- $\kappa \mathrm{B}$ in the lung. Fluorescence intensity of mtROS was increased in cytoplasm of BAL cells from $A a$-exposed mice, as seen in that of $A f$-exposed mice. Dexamethasone significantly reduced the $A a$-induced generation of mtROS in these cells. However, dexamethasone failed to reduce the $A f$-induced generation of mtROS (online supplementary figure S12A, B). Furthermore, levels of NF- $\mathrm{kB}$ p65 in nuclear protein extracts of lung tissues were significantly increased after exposure to $A f$ or $A a$, but levels of NF- $\mathrm{KB}$ p 65 in cytosolic protein extracts of lung tissues were remarkably decreased after exposure to $A f$ and $A a$. Interestingly, while dexamethasone significantly reduced the $A a$-induced increases in the nuclear translocation of

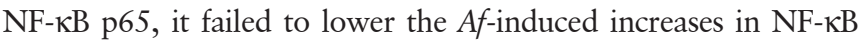
p65 in nuclear protein extracts (online supplementary figure S12CF). These findings suggest that the difference in the ability of dexamethasone to control inflammation in the two mouse models is in part dependent on whether CS can regulate fungi-induced generation of mtROS.

\section{DISCUSSION}

In this study, assembly/activation of NLRP3 inflammasome was increased in the lung of Af-exposed mice. Elevation of NLRP3 inflammasome assembly/activation was also observed in Af-stimulated murine and human EpCs. Similarly, expression of NLRP3 was increased in lung tissue from patients with ABPA. Importantly, neutralisation of NLRP3-derived IL-1 $\beta$ alleviated various features of $A f$-induced allergic inflammation. Furthermore, inhibition of PI $3 \mathrm{~K}-\delta$ improved $A f$-induced allergic inflammation through modulating NLRP3 inflammasome assembly/activation, especially in EpCs. This modulatory role of PI $3 \mathrm{~K}-\delta$ was mediated through the regulation of mtROS generation. NLRP3 inflammasome was also implicated in $A a$-induced allergic lung inflammation, which was improved by PI $3 \mathrm{~K}-\delta$ blockade. These findings demonstrate for the first time that fungi-induced assembly/activation of NLRP3 inflammasome in bronchial EpCs may be modulated by PI $3 \mathrm{~K}-\delta$, which is mediated partly through the regulation of $\mathrm{mtROS}$ generation.

Cytokine imbalance is important in chronic lung disorders. ${ }^{35}$ Particularly, IL-1 $\beta$, which activates many inflammatory genes implicated in allergic inflammation, ${ }^{36}$ is increased in asthmatic airways, and blockade of IL-1 $\beta$ ameliorated allergic lung inflammation in a murine model. ${ }^{37}$ NLRP3 inflammasome has been known to be implicated in allergic inflammatory processes as well as antifungal defence through IL-1 $\beta .^{8}{ }^{18}$ Nevertheless, the role of NLRP3 inflammasome in allergic lung inflammation is still unclear. While several reports favour the critical involvement of NLRP3 inflammasome in allergic lung inflammation, ${ }^{8} 11$ conflicting data also exist. ${ }^{19}{ }^{20}$ However, given that upregulation of NLRP3 inflammasome is observed in patients with asthma having neutrophilic inflammation, ${ }^{38}$ the role of NLRP3 inflammasome in allergic inflammation might be different according to underlying biological processes. In our study, $A f$ exposure resulted in the increases of NLRP3, caspase- 1 and IL-1 $\beta$, and physical associations between NLRP3 inflammasome components in lung tissues. It was supported by the observation that immunofluorescence intensities of NLRP3 components and their cytoplasmic co-localisations were increased in BAL cells. Increases in immunofluorescence intensities and co-localisation of NLRP3 and caspase- 1 were also observed in lung tissues. Especially, NLRP3 expression is increased in lung tissues from patients with ABPA, one proposed asthma endotype characterised by severe allergic inflammation associated with $A f \cdot{ }^{39}$ Furthermore, $A f$ stimulation led to the increases in immunofluorescence intensities and their cytoplasmic co-localisations of NLRP3 inflammasome components as well as ASC oligomerisation in murine and human EpCs. Notably, selective inhibition of NLRP3 inflammasome led to the decrease of IL-1 $\beta$ in the lung of $A f$-exposed mice and neutralisation of IL-1 $\beta$ improved numerous features of $A f$-induced allergic lung inflammation. Similarly, NLRP3 inflammasome blockade ameliorated $A a$-induced increases of IL-1 $\beta$ and allergic inflammation in the lung. Taken together, NLRP3 inflammasome may play a pivotal role in fungi-induced allergic lung inflammation.

NLRP3 inflammasome senses and reacts to diverse stimuli through various mechanisms. ${ }^{5}$ However, scarce information exists on the regulatory mechanism of NLRP3 inflammasome activation against fungal exposure, particularly in allergic inflammation. PI3K- $\delta$ plays a key role in regulation of immune processes through activating immune cells and trafficking inflammatory cells. ${ }^{40}$ PI $3 \mathrm{~K}-\delta$ is also activated in response to fungal exposure and plays a role in the regulation of ER stress, thereby being crucially implicated in fungal allergic inflammation. ${ }^{22}$ ER stress is one of the triggers of NLRP3 inflammasome activation. ${ }^{10} 23$ In this study, PI3K- $\delta$ inhibition attenuated $A f$-induced airway inflammatory cell infiltrations, AHR and elevations of pulmonary Th2 cytokines and serum total and $A f$-specific IgE, highlighting the pivotal role of PI3K- $\delta$ in $A f$-induced allergic inflammation. Importantly, $A f$-induced increases of NLRP3, active caspase- 1 and IL- $1 \beta$ in the lung were significantly reduced by IC 87114 . Furthermore, $A$-exposed $\mathrm{p} 110 \delta \mathrm{KO}$ mice showed the lower levels of NLRP3, caspase- 1 and IL-1 $\beta$ in the lung compared with Af-exposed WT mice. The regulatory role of PI3K- $\delta$ on NLRP3 inflammasome was further verified by the IP assay. Af-induced increases in the immunofluorescence intensities of NLRP3 inflammasome components and their cytoplasmic co-localisations in BAL cells were also reduced by IC87114. In $A f$-stimulated murine and human EpCs, Af-induced increased ASC oligomerisation was reduced by IC87114. Moreover, $A f$-induced increases in the immunofluorescence intensities and cytoplasmic co-localisations of NLRP3 inflammasome components in EpCs were reduced by IC87114 or PI3K- $\delta$ siRNA. $A f$-induced increases of NLRP3 and IL-1 $\beta$ were also reduced by IC 87114 or PI3K- $\delta$ siRNA in these cells. In $A a$-induced allergic inflammation, IC87114 attenuated the $A a$-induced increases of inflammatory cell infiltrations, Th2 cytokines and IL-1 $\beta$ in the lung. These findings imply that PI $3 \mathrm{~K}-\delta$ is critically implicated in fungi-induced allergic lung inflammation through regulation of NLRP3 inflammasome in airway epithelium.

Oxidative stress is one of the most characteristic features of chronic airway disorders ${ }^{41}$ and mtROS are a common molecular platform in NLRP3 inflammasome activation. ${ }^{59342}$ Since most of the intracellular ROS originate from mitochondria, we examined roles of mtROS in this study. Fluorescence intensity of mtROS was increased in the cytoplasm of BAL cells, particularly macrophages and eosinophils, from $A f$-exposed and $A a$-exposed mice. A potent mtROS scavenger alleviated $A f$-induced mtROS 
generation in these cells. Importantly, the mtROS scavenger reduced the $A f$-induced increase in IL- $1 \beta$, and that improved various features of allergic lung inflammation in $A f$-exposed mice. Recently, we reported that mtROS generation in airway epithelium is fundamental in allergic inflammation ${ }^{11}$ and PI3K- $\delta$ regulates mtROS production in airway EpCs under fungal exposure. ${ }^{22}$ Likewise, in the present study, $A f$-induced increases in the fluorescence intensity of mtROS were lowered by IC87114 in murine and human EpCs as well as BAL cells from Af-exposed mice. These findings suggest that PI3K- $\delta$ modulates NLRP3 inflammasome, partly through inducing mtROS in airway epithelium, thereby contributing to fungi-induced allergic inflammation.

CS is the most powerful anti-inflammatory agent available for immune-mediated disorders. However, $5 \sim 10 \%$ of patients with asthma have severe disorders accounting for more than $50 \%$ of the total healthcare cost, and CS resistance is a common feature for severe asthma. ${ }^{34}{ }^{43}$ Fungi-associated asthma endotype may present more severe disease with frequent exacerbations and eosinophilic inflammation. ${ }^{39}$ Among fungi implicated in severe asthma, $A f$ can tolerate body temperature and grow in the airways, thereby colonising and inducing potent immune responses. Therefore, it has been suggested that the most severe form of allergic fungal disease frequently involves $A f \cdot{ }^{44}$ In the current study, whereas dexamethasone improved various features of $A a$-induced allergic lung inflammation, it failed to alleviate Af-induced inflammatory cell infiltration, AHR and elevations of Th2 cytokines in the lung. Dexamethasone also reduced $A a$-induced elevations of NLRP3, caspase- 1 and IL-1 $\beta$ in the lung. However, dexamethasone failed to reduce $A f$-induced elevations of NLRP3, caspase-1 and IL-1 $\beta$. Additionally, the difference in the ability of dexamethasone to control inflammation in the two mouse models may be partly dependent on whether CS can regulate fungi-induced generation of mtROS. In fact, oxidative stress is closely involved in the development of CS resistance in severe asthma and chronic obstructive pulmonary disease. ${ }^{34}$ Moreover, generation of mtROS is closely implicated in CS-resistant inflammation in the lung. ${ }^{22}$ Further, NLRP3 inflammasome has been suggested to be involved in severe allergic lung inflammation, particularly in neutrophilic inflammation ${ }^{113845}$ that is generally regarded as an important phenotype for severe asthma. Moreover, NLRP3 activation has been proposed to be associated with increased CS resistance in certain leukaemia cells. ${ }^{46}$ Therefore, pulmonary mtROS generation and the related activation of NLRP3 inflammasome may be linked to fungi-induced CS resistance associated with specific fungal species including $A f$.

In summary, we demonstrated that NLRP3 inflammasome is involved in fungi-induced allergic lung inflammation and may be linked to CS resistance in $A f$-induced allergic inflammation. Furthermore, PI3K- $\delta$ is critically implicated in fungi-induced allergic lung inflammation through regulation of NLRP3 inflammasome assembly/activation in airway epithelium, which is mediated partly through the regulation of mtROS generation. This suggests that inhibition of PI3K- $\delta$ may have potential for treating fungi-induced severe allergic lung inflammation in humans.

\section{Author affiliations}

${ }^{1}$ Department of Internal Medicine, Research Center for Pulmonary Disorders, Chonbuk National University Medical School, Jeonju, South Korea

${ }^{2}$ Research Institute of Clinical Medicine of Chonbuk National University, Biomedical Research Institute of Chonbuk National University Hospital, Jeonju, South Korea ${ }^{3}$ Department of Immunology, Chonbuk National University Medical School, Jeonju, South Korea

${ }^{4}$ Department of Urology, Chonbuk National University Medical School, Jeonju, South Korea
}

${ }^{5}$ Division of Allergy and Immunology, Internal Medicine, Morsani College of Medicine, University of South Florida, Tampa, Florida, USA

${ }^{6}$ Department of Product Strategy and Development, LG Life Sciences Ltd, Seoul, South Korea

Contributors JSJ interpreted data and wrote the manuscript. KBL, DIK and HJP conducted experiments and performed analysis. H-KL, HJK, SHC, NK and SHK reviewed and edited the manuscript. SRK and YCL designed research, interpreted data, and edited the manuscript.

Funding This work was supported by the Basic Science Research Program through the National Research Foundation of Korea (NRF) funded by the Ministry of Science, ICT and Future Planning (NRF-2014R1A2A1A01002823; Y.C.L.).

Competing interests None declared.

Ethics approval Institutional Review Board of the Biomedical Research Institute of Chonbuk National University Hospital (IRB file No. 2013-11-007-001).

Provenance and peer review Not commissioned; externally peer reviewed.

Open access This is an open access article distributed in accordance with the Creative Commons Attribution Non Commercial (CC BY-NC 4.0) license, which permits others to distribute, remix, adapt, build upon this work non-commercially, and license their derivative works on different terms, provided the original work is properly cited and the use is non-commercial. See: http://creativecommons.org/ licenses/by-nc/4.0/

(c) Article author(s) (or their employer(s) unless otherwise stated in the text of the article) 2018. All rights reserved. No commercial use is permitted unless otherwise expressly granted.

\section{REFERENCES}

1 Holgate ST. Innate and adaptive immune responses in asthma. Nat Med 2012;18:673-83.

2 Takeuchi O, Akira S. Pattern recognition receptors and inflammation. Cell 2010;140:805-20.

3 Lambrecht BN, Hammad H. The immunology of asthma. Nat Immunol 2015;16:45-56

4 Kanneganti TD, Lamkanfi M, Núñez G. Intracellular NOD-like receptors in host defense and disease. Immunity 2007:27:549-59.

5 Guo H, Callaway JB, Ting JP. Inflammasomes: mechanism of action, role in disease, and therapeutics. Nat Med 2015:21:677-87.

6 Schroder K, Tschopp J. The inflammasomes. Cell 2010;140:821-32.

7 Franchi L, Muñoz-Planillo R, Núñez G. Sensing and reacting to microbes through the inflammasomes. Nat Immunol 2012;13:325-32.

8 Besnard AG, Guillou N, Tschopp J, et al. NLRP3 inflammasome is required in murine asthma in the absence of aluminum adjuvant. Allergy 2011;66:1047-57.

9 Zhou R, Yazdi AS, Menu P, et al. A role for mitochondria in NLRP3 inflammasome activation. Nature 2011:469:221-5

10 Bronner DN, Abuaita BH, Chen X, et al. Endoplasmic reticulum stress activates the inflammasome via NLRP3- and caspase-2-driven mitochondrial damage. Immunity 2015:43:451-62

11 Kim SR, Kim DI, Kim SH, et al. NLRP3 inflammasome activation by mitochondrial ROS in bronchial epithelial cells is required for allergic inflammation. Cell Death Dis 2014:5:e1498.

12 Denning DW, O'Driscoll BR, Hogaboam CM, et al. The link between fungi and severe asthma: a summary of the evidence. Eur Respir J 2006:27:615-26.

13 O'Driscoll BR, Powell G, Chew F, et al. Comparison of skin prick tests with specific serum immunoglobulin $\mathrm{E}$ in the diagnosis of fungal sensitization in patients with severe asthma. Clin Exp Allergy 2009;39:1677-83.

14 Knutsen AP, Bush RK, Demain JG, et al. Fungi and allergic lower respiratory tract diseases. J Allergy Clin Immunol 2012;129:280-91.

15 Fairs A, Agbetile J, Hargadon B, et al. IgE sensitization to Aspergillus fumigatus is associated with reduced lung function in asthma. Am J Respir Crit Care Med 2010;182:1362-8

16 Denning DW, Pashley C, Hartl D, et al. Fungal allergy in asthma-state of the art and research needs. Clin Trans/ Allergy 2014;4:14

17 Denning DW, O'Driscoll BR, Powell G, et al. Randomized controlled trial of oral antifungal treatment for severe asthma with fungal sensitization: The Fungal Asthma Sensitization Trial (FAST) study. Am J Respir Crit Care Med 2009;179:11-18.

18 Gross 0 , Poeck H, Bscheider M, et al. Syk kinase signalling couples to the Nlrp3 inflammasome for anti-fungal host defence. Nature 2009;459:433-6.

19 Kool M, Willart MA, van Nimwegen M, et al. An unexpected role for uric acid as an inducer of $T$ helper 2 cell immunity to inhaled antigens and inflammatory mediator of allergic asthma. Immunity 2011;34:527-40.

20 Allen IC, Jania CM, Wilson JE, et al. Analysis of NLRP3 in the development of allergic airway disease in mice. J Immunol 2012:188:2884-93.

21 Kim SR, Lee KS, Park HS, et al. HIF-1 $\alpha$ inhibition ameliorates an allergic airway disease via VEGF suppression in bronchial epithelium. Eur J Immunol 2010;40:2858-69. 
22 Lee KS, Jeong JS, Kim SR, et al. Phosphoinositide 3-kinase- $\delta$ regulates fungusinduced allergic lung inflammation through endoplasmic reticulum stress. Thorax 2016;71:52-63.

23 Menu P, Mayor A, Zhou R, et al. ER stress activates the NLRP3 inflammasome via an UPR-independent pathway. Cell Death Dis 2012;3:e261.

24 Oczypok EA, Milutinovic PS, Alcorn JF, et al. Pulmonary receptor for advanced glycation end-products promotes asthma pathogenesis through IL-33 and accumulation of group 2 innate lymphoid cells. J Allergy Clin Immunol 2015; 136:747-56

25 Kwak YG, Song CH, Yi HK, et al. Involvement of PTEN in airway hyperresponsiveness and inflammation in bronchial asthma. J Clin Invest 2003;111:1083-92.

26 De Francesco EM, Lappano R, Santolla MF, et al. HIF-1 $\alpha /$ GPER signaling mediates the expression of VEGF induced by hypoxia in breast cancer associated fibroblasts (CAFs). Breast Cancer Res 2013;15:R64.

27 Lopez-Castejon G, Luheshi NM, Compan V, et al. Deubiquitinases regulate the activity of caspase- 1 and interleukin- $1 \beta$ secretion via assembly of the inflammasome. J Biol Chem 2013;288:2721-33.

28 Segal BH. Aspergillosis. N Engl J Med 2009;360:1870-84.

29 Stevens DA, Moss RB, Kurup VP, et al. Allergic bronchopulmonary aspergillosis in cystic fibrosis--state of the art: Cystic Fibrosis Foundation Consensus Conference. Clin Infect Dis 2003;37 Suppl 3(Suppl 3):S225-S264.

30 Bauernfeind FG, Horvath G, Stutz A, et al. Cutting edge: NF-kappaB activating pattern recognition and cytokine receptors license NLRP3 inflammasome activation by regulating NLRP3 expression. J Immunol 2009;183:787-91.

31 Osherov N. Interaction of the pathogenic mold Aspergillus fumigatus with lung epithelial cells. Front Microbio/ 2012:3:346.

32 Coll RC, Robertson AA, Chae JJ, et al. A small-molecule inhibitor of the NLRP3 inflammasome for the treatment of inflammatory diseases. Nat Med 2015;21:248-55.

33 Nakahira K, Haspel JA, Rathinam VA, et al. Autophagy proteins regulate innate immune responses by inhibiting the release of mitochondrial DNA mediated by the NALP3 inflammasome. Nat Immunol 2011:12:222-30.
34 Barnes PJ. Corticosteroid resistance in patients with asthma and chronic obstructive pulmonary disease. J Allergy Clin Immunol 2013;131:636-45.

35 Barnes PJ. The cytokine network in asthma and chronic obstructive pulmonary disease. J Clin Invest 2008;118:3546-56.

36 Dinarello CA. Immunological and inflammatory functions of the interleukin-1 family. Annu Rev Immunol 2009;27:519-50.

37 Johnson VJ, Yucesoy B, Luster MI. Prevention of IL-1 signaling attenuates airway hyperresponsiveness and inflammation in a murine model of toluene diisocyanateinduced asthma. J Allergy Clin Immunol 2005;116:851-8.

38 Simpson JL, Phipps S, Baines KJ, et al. Elevated expression of the NLRP3 inflammasome in neutrophilic asthma. Eur Respir J 2014;43:1067-76.

39 Lötvall J, Akdis CA, Bacharier LB, et al. Asthma endotypes: a new approach to classification of disease entities within the asthma syndrome. J Allergy Clin Immunol 2011;127:355-60.

40 Park SJ, Min KH, Lee YC. Phosphoinositide 3-kinase delta inhibitor as a novel therapeutic agent in asthma. Respirology 2008;13:764-71.

41 Ciencewicki J, Trivedi S, Kleeberger SR. Oxidants and the pathogenesis of lung diseases. J Allergy Clin Immunol 2008;122:456-68.

42 Dostert C, Pétrilli V, Van Bruggen R, et al. Innate immune activation through Nalp3 inflammasome sensing of asbestos and silica. Science 2008;320:674-7.

43 Chung KF, Wenzel SE, Brozek JL, et al. International ERS/ATS guidelines on definition, evaluation and treatment of severe asthma. Eur Respir 」 2014:43:343-73.

44 Agarwal R, Gupta D. Severe asthma and fungi: current evidence. Med Mycol 2011:49(Suppl 1):S150-7.

$45 \mathrm{Kim} \mathrm{HY}$, Lee HJ, Chang YJ, et al. Interleukin-17-producing innate lymphoid cells and the NLRP3 inflammasome facilitate obesity-associated airway hyperreactivity. Nat Med 2014;20:54-61.

46 Paugh SW, Bonten EJ, Savic D, et al. NALP3 inflammasome upregulation and CASP1 cleavage of the glucocorticoid receptor cause glucocorticoid resistance in leukemia cells. Nat Genet 2015;47:607-14. 